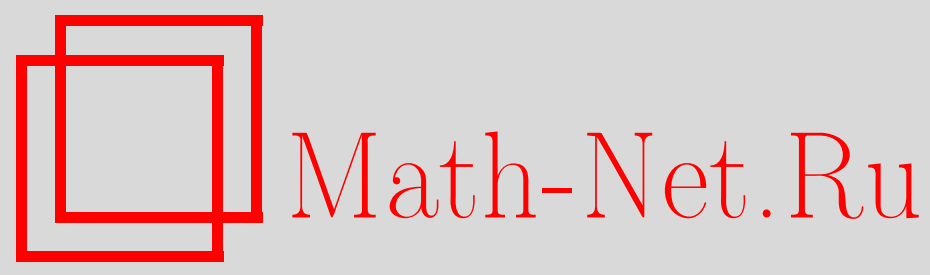

П. А. Бородин, О линейности оператора метрического проектирования на чебышевские подпространства в пространствах $L_{1}$ и $C$, Матем. заметки, 1998 , том 63, выпуск $6,812-$ 820

DOI: https://doi.org/10.4213/mzm1351

Использование Общероссийского математического портала Math-Net.Ru подразумевает, что вы прочитали и согласны с пользовательским соглашением http://www.mathnet.ru/rus/agreement

Параметры загрузки:

IP : 52.90 .164 .192

26 апреля 2023 г., $15: 13: 46$

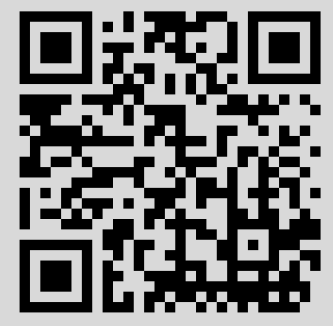




\section{О ЛИНЕЙНОСТИ ОПЕРАТОРА МЕТРИЧЕСКОГО ПРОЕКТИРОВАНИЯ НА ЧЕБЫШЕВСКИЕ ПОДПРОСТРАНСТВА В ПРОСТРАНСТВАХ $L_{1}$ И $C$}

\section{П. А. Бородин}

Для чебьшевского подпространства $Y$ в банаховом пространстве $X$ определен однозначный оператор метрического проектирования $P_{Y}: X \rightarrow Y$, сопоставляющий каждому $x \in X$ ближайший к нему элемент $y \in \mathrm{Y}$. Пусть $M$ - произвольное множество, $\mu-\sigma$-конечная мера на некоторой $\sigma$-алгебре $\Sigma$ подмножеств $M$. В работе полностью описаны чебышевские подпространства $Y \subset L_{1}(M, \Sigma, \mu)$ с линейным оператором $P_{Y}$ (в случае пространства $L_{1}[0,1]$ это было сделано П. Моррисом в 1980 году). Указан шшрокий класс чебышевских подпространств в $L_{1}(M, \Sigma, \mu)$ с, вообще говоря, нелинейньм оператором $P_{Y}$. Доказано, что оператор $P_{Y}$ для нетривиального собственного чебышевского подпространства $Y \subset C[K](K$ - компакт) линеен тогда и только тогда, когда $Y$ имеет коразмерность 1 в $C[K]$.

Библиографоия: 9 названий.

0. Введение. Пусть $X$ - банахово пространство, $Y$ - его (замкнутое линейное) подпространство, $\rho(x, Y)=\inf \{\|x-y\|: y \in Y\}-$ расстояние от элемента $x \in X$ до $Y$, $P_{Y}(x)=\{y \in Y:\|x-y\|=\rho(x, Y)\}-$ множество ближайших к $x$ элементов из $Y$.

Подпространство $Y$ называется чебыиевским, если для каждого $x \in X$ множество $P_{Y}(x)$ состоит ровно из одного элемента. Другими словами, для каждого $x \in X$ имеет место существование и единственность элемента наилучшего приближения при апшроксимации элементами подпространства $Y$. Возникающий при этом оператор метрического проектирования $P_{Y}: X \rightarrow Y$, сопоставляюший каждому элементу $x \in X$ его элемент наилучшего приближения $P_{Y}(x) \in Y$, не является, вообще говоря, ни линейным, ни даже непрерьвньм [1], [2].

Цель настоящей работы состоит в описании тех чебьшевских подпространств $Y$ в пространствах $L_{1}$ и $C$, для которых оператор $P_{Y}$ линеен (теоремы 1 и 3 ). Кроме того, в пространстве $L_{1}$ мы укажем достаточно широкий класс чебьшевских подпространств $Y$ c, вообще говоря, нелинейным оператором $P_{Y}$ (теорема 2 ).

Отметим, что теорема 1 аналогична теореме П. Морриса [3], описавшего чебьшевские подпространства с линейным оператором метрического проектирования в пространстве $L_{1}[0,1]$.

Основным инструментом нашего исследования будут так назьваемые квазиортогональные множества.

Работа выполнена при поддержке Российского фонда фундаментальных исследований, грант № 96-01-01366. 
ОПРЕДЕЛЕНИЕ. Квазиортогональным множеством $Q(Y)=Q(Y, X)$ к подпространству $Y$ в пространстве $X$ назовем совокупность всех элементов $n \in X$, для которых $P_{Y}(n) \ni 0$.

Этот объект впервые появляется в работах Э. Чини и Д. Вульберта [2] и И. Зингера [1] (термин квазиортогональное множсество предложил Е. П. Долженко). В случае гильбертова пространства $X$ каждый элемент $n \in Q(Y)$ ортогонален подпространству $Y$ в обычном смысле, а само множество $Q(Y)$ является ортогональньм дополнением к $Y$.

Приведем основные свойства квазиортогональных множеств.

Множество, квазиортогональное к подпространству, всегда замкнуто и является двусторонним конусом: если $n \in Q(Y)$, то $\lambda n \in Q(Y)$ для всякого числа $\lambda$. Очевидно, в простейшем случае $Y=X$ множество $Q(Y)$ совпадает с нулевьп подпространством, и обратно, $Q(\{0\})=X$.

ЛЕмма 1 [2]. Подпространство $Y$ является чебышевским тогда и только тогда, когда выполнены два условия:

1) $Q(Y)+Y=X$;

2) для любого $n \in Q(Y) \quad P_{Y}(n)=\{0\}$.

При этом 1) эквивалентно существованию, а 2) - единственности әлемента наилучшего приближения.

Из леммы 1 непосредственно вытекает

Лемма 2 [1]. Оператор $P_{Y}$ метрического проектирования на чебышевское подпространство $Y$ линеен тогда и только тогда, когда $Q(Y)$ - линейное подпространство.

Отметим следующий частный случай линейности $P_{Y}$.

Лемма 3. Если чебъиевское подпространство $Y$ имеет линейную коразмерность 1 в $X$, то $P_{Y}$ - линейный оператор.

ДокАЗАтЕльство. Действительно, $Q(Y) \neq\{0\}$ согласно лемме 1. С другой стороны, если $Q(Y)$ содержит два линейно независимых элемента $p$ и $q$, то для некоторого скаляра $\lambda$ имеем $y:=p-\lambda q \in Y, y \neq 0$. Отсюда $p-y=\lambda q \in Q(Y)$, и у элемента $p$ в подпространстве $Y$ оказывается по крайней мере два элемента наилучшего приближения -0 и $y$. Это невозможно, поскольку $Y$ - чебьшевское подпространство. Итак, $Q(Y)$ - одномерное подпространство, и по лемме 2 оператор $P_{Y}$ линеен.

В то же время условие 2) в лемме 1 можно заменить условием линейности множества, квазиортогонального к подпространству $Y$.

Лемма 4. Если $Q(Y)$ - подпространство и $Q(Y)+Y=X$, то $Y$ - чебьшевское подпространство.

ДокАЗАТЕльство. В самом деле, предположим, что у какого-то $x \in X$ элемент наилучшего приближения не единственньй. Тогда $x=n_{1}+y_{1}=n_{2}+y_{2}$, где $n_{1}, n_{2} \in Q(Y)$, $y_{1}, y_{2} \in Y$ и $y_{1} \neq y_{2}$. Отсюда $n_{1}-n_{2}=y_{2}-y_{1} \neq 0$. Так как $Q(Y)$ - подпространство, то $Q(Y) \ni n_{1}-n_{2}=y_{2}-y_{1} \in Y$, так что множество $Y \cap Q(Y)$ содержит ненулевой элемент, что невозможно. Лемма 4 доказана. 
В конце этого пункта докажем утверждение, позволяющее непосредственно определять множество $Q(Y)$.

Пусть $Y^{\perp}=\left\{f \in X^{*}: f(y)=0 \quad \forall y \in Y\right\}$ - аннулятор подпространства $Y$ в сопряженном пространстве $X^{*}$.

Лемма 5. $Q(Y)=\left\{n \in X:\right.$ существует ненулевой функиионал $f \in Y^{\perp}$, для которого $f(n)=\|f\| \cdot\|n\|\}$.

ДокАЗАТЕльСтво. Действительно, для всякого элемента $n$ и всякого $y \in Y$ имеем

$$
\|n-y\| \geqslant \frac{|f(n-y)|}{\|f\|}=\frac{|f(n)|}{\|f\|}=\|n\|,
$$

так что $0 \in P_{Y}(n)$. Обратно, пусть $n \in Q(Y)$. Обозначим через $\langle n\rangle$ одномерное подпространство, порожденное вектором $n$, и рассмотрим функционал $f_{0}$ на пространстве $Y \oplus\langle n\rangle$, определяемьй равенством $f_{0}(y+\lambda n)=\lambda$. Поскольку

$$
|\lambda|=\frac{\|\lambda n\|}{\|n\|} \leqslant \frac{\|y+\lambda n\|}{\|n\|},
$$

норма функционала $f_{0}$ равна $1 /\|n\|$. По теореме Хана-Банаха $f_{0}$ можно, не увеличивая нормы, линейно продолжить на все пространство $X$, в результате получится функционал $f \in Y^{\perp}$, для которого $f(n)=1=\|n\| \cdot\|f\|$.

Лемму 5 можно интерпретировать следующим образом: для того чтобы получить $Q(Y)$, необходимо взять все функционалы из $Y^{\perp}$, достигающие своей нормы, а затем все элементы из $X$, на которых эта норма достигается. Совокупность всех таких элементов и есть $Q(Y)$.

1. Пространство $L_{1}$. Далее через $L_{1}(M)=L_{1}(M, \Sigma, \mu)$ обозначим пространство вешественнозначных функций, определенных на множестве $M$ произвольной природы и суммируемых на $M$ по мере $\mu$, заданной на $\sigma$-алгебре $\Sigma$ подмножеств этого множества. Для каждой функции $x \in L_{1}(M)$ определим ее носитель supp $x:=\{t \in M: x(t) \neq 0\}$. Разумеется, supp $x$ определен с точностью до множества меры 0.

Мера $\mu$ предполагается $\sigma$-конечной. В этом случае сопряженным к $L_{1}(M)$ является пространство $L_{\infty}(M)$ функций, существенно ограниченных на $M$ (см., например, [4]). Именно, функция $f \in L_{\infty}(M)$ определяет функционал $f: x \mapsto \int f x d \mu\left(x \in L_{1}(M)\right)$. Этот функционал достигает своей нормы в том и только том случае, когда функция $f$ сама достигает своей нормы $\|f\|=\operatorname{ess} \sup \{|f(t)|: t \in M\}$ на множестве положительной меры, которое обозначим через $m(f)$. При этом норма достигается на любой функции $n(t) \in L_{1}(M)$, носитель которой supp $n \subseteq m(f)$ и которая удовлетворяет равенству $\operatorname{sign} n(t)=f(t) /\|f\|$ при почти всех $t \in \operatorname{supp} n$. Такие функции $n$ назовем подчиненными функционалу $f$. Все эти функции $n$, подчиненные каждая своему функционалу $f \in Y^{\perp}$, и составляют квазиортогональное множество $Q(Y)$ к подпространству $Y$ (см. лемму 5$)$. О чебьшевских подпространствах в $L_{1}(M)$ см. [1], [5].

ЗАмЕчАниЕ 1. Отметим, что чебьшевские подпространства конечной размерности или коразмерности в $L_{1}(M)$ полностью описаны Р. Фелпсом [6] и А. Л. Гаркави [7]. В утверждениях, доказываемых ниже, фигурируют чебьшевские подпространства, вообще говоря, бесконечной размерности и коразмерности.

Следующая теорема характеризует чебьшевские подпространства $Y \subset L_{1}(M)$, у которых оператор метрического проектирования $P_{Y}$ линеен. 
Tеорема 1. Подпространство $Y \subset L_{1}(M)$ является чебышевским подпространством с линейным оператором метрического проектирования тогда и только тогда, когда существуют разбиение множества $M$ на два непересекающихся $\mu$-измеримых множества $M_{1}$ и $M_{2}$ и линейный оператор $A: L_{1}\left(M_{1}\right) \rightarrow L_{1}\left(M_{2}\right)$, строго уменьиаюший норму каждого әлемента $(\|A(y)\|<\|y\|)$, такие, что $Y=$ $\left\{y \in L_{1}(M):\left.y\right|_{M_{2}}=A\left(\left.y\right|_{M_{1}}\right)\right\}$, где $\left.y\right|_{N}-$ сужение функиии у на множество $N \subseteq M$.

ЗАмечаниЕ 2. В условиях этой теоремы $Q(Y)$ состоит из всех функций $x \in L_{1}(M)$, равных 0 почти всюду на множестве $M_{1}$, а оператор метрического проектирования действует так: $P_{Y}(x)=\left.x\right|_{M_{1}}+A\left(\left.x\right|_{M_{1}}\right)$.

Для пространства $L_{1}[0,1]$ эта теорема доказана в [3].

ДокАЗАТЕЛЬСтво. Для простоты записи обозначим через $L_{1}(N)$ подпространство всех функций из $L_{1}(M)$, равных 0 почти всюду (по мере $\mu$ ) вне множества $N \subset M$.

Необходимость. Пусть $n \in Q(Y)$. Возьмем произвольную функцию $x \in L_{1}(\operatorname{supp} n)$. Очевидно, найдутся функции $n_{1}, n_{2} \in L_{1}(M)$ такие, что $\operatorname{sign} n_{1} \equiv \operatorname{sign} n_{2} \equiv \operatorname{sign} n$ и $x \equiv n_{1}-n_{2}$. Поскольку $n_{1}, n_{2} \in Q(Y)$ (они подчинены тому же функционалу, что и $n)$ и $Q(Y)$ - линейное подпространство (по лемме 2 ), то $x \in Q(Y)$. Таким образом, $L_{1}(\operatorname{supp} n) \subseteq Q(Y)$.

Положим

$$
M_{2}=\bigcup_{n \in Q(Y)} \operatorname{supp} n
$$

Пусть $M_{2}=\cup M_{2 k}$, где $\mu\left(M_{2 k}\right)<\infty, k=1,2, \ldots$ (мера $\mu \sigma$-конечна). Для каждого $k$ найдется не более чем счетное число функций $n_{k l} \in Q(Y)$ таких, что

$$
\mu\left(M_{2 k} \backslash \bigcup_{l} \operatorname{supp} n_{k l}\right)=0
$$

В этом случае всякую функцию $x_{k} \in L_{1}\left(M_{2 k}\right)$ можно с любой точностью приблизить (в норме $L_{1}$ ) функцией $y_{k}$ с носителем на конечном объединении $\cup \operatorname{supp} n_{k l}$. Из сказанного выше следует, что $y_{k} \in Q(Y)$. Всякую функцию $x \in L_{1}\left(M_{2}\right)$ можно с любой точностью приблизить конечной суммой функций $x_{k}$, а значит, и конечной суммой функций $y_{k}$, т.е. просто функцией из $Q(Y)$. Пользуясь замкнутостью $Q(Y)$, получаем $L_{1}\left(M_{2}\right) \subseteq Q(Y)$. Обратное включение вытекает из определения множества $M_{2}$. Итак, $Q(Y)=L_{1}\left(M_{2}\right)$.

Положим $M_{1}=M \backslash M_{2}$. Если $\mu\left(M_{1}\right)=0$, то $Q(Y)=L_{1}(M), Y=\{0\}$ и искомый оператор $A$ нулевой.

Пусть теперь $\mu\left(M_{1}\right)>0$. Всякий элемент $y \in Y$ однозначно представим в виде $y=y_{1}+y_{2}$, где $y_{k} \in L_{1}\left(M_{k}\right), k=1,2$. При этом функции $y_{1}$ пробегают все подпространство $L_{1}\left(M_{1}\right)$, иначе $Y+Q(Y)=Y+L_{1}\left(M_{2}\right) \neq L_{1}(M)$ и $Y$ - не чебышевское подпространство согласно лемме 1 . Кроме того, $y_{1} \neq 0$ при $y \neq 0$ (иначе $Y \cap Q(Y) \neq\{0\}$ ), и $y_{2}$ линейно зависит от $y_{1}$. С другой стороны, $y_{2} \in Q(Y)$, следовательно, при $y \neq 0$ выполняется строгое неравенство $\left\|y_{1}\right\|=\left\|y_{2}-y\right\|>\left\|y_{2}\right\|$ (строгость объясняется тем, что 0 - единственный элемент наилучшего приближения для $y_{2}$ в подпространстве $Y$ ). Таким образом, $y_{2}=A\left(y_{1}\right)$, где $A: L_{1}\left(M_{1}\right) \rightarrow L_{1}\left(M_{2}\right)$ - линейньй оператор, строго уменьшающий норму: $\left\|A y_{1}\right\|<\left\|y_{1}\right\|$. 
Достаточность. Пусть подпространство $Y$ задано множествами $M_{1}, M_{2}$ и оператором $A$. Докажем, что $Q(Y)=L_{1}\left(M_{2}\right)$. Действительно, для любых $n \in L_{1}\left(M_{2}\right)$ и $y=y_{1}+y_{2} \in Y$ имеем

$$
\begin{aligned}
\|n-y\| & =\int_{M}\left|n-y_{1}-y_{2}\right| d \mu=\int_{M_{1}}\left|y_{1}\right| d \mu+\int_{M_{2}}\left|n-y_{2}\right| d \mu \\
& \geqslant \int_{M_{1}}\left|y_{1}\right| d \mu+\int_{M_{2}}|n| d \mu-\int_{M_{2}}\left|y_{2}\right| d \mu=\left\|y_{1}\right\|+\|n\|-\left\|y_{2}\right\|>\|n\|,
\end{aligned}
$$

поскольку $\left\|y_{2}\right\|=\left\|A\left(y_{1}\right)\right\|<\left\|y_{1}\right\|$. Мы показали, что $L_{1}\left(M_{2}\right) \subseteq Q(Y)$. Если для некоторого $n \in Q(Y)$ вьполняется неравенство $\mu\left(\operatorname{supp} n \cap M_{1}\right)>0$, то, положив $n_{1}=\left.n\right|_{M}$, получим $n_{1} \in Q(Y)$ ( $n_{1}$ подчинен тому же функционалу из $Y^{\perp}$, что и $\left.n\right)$. Но элемент $y=n_{1}+A\left(n_{1}\right)$ ближе к $n_{1}$, чем нулевой элемент: $\rho\left(n_{1}, 0\right)=\left\|n_{1}\right\|>\left\|A\left(n_{1}\right)\right\|=\rho\left(n_{1}, y\right)$. Это противоречие показывает, что для всякого $n \in Q(Y)$ равенство $\mu\left(\operatorname{supp} n \cap M_{1}\right)=0$ имеет место, а значит, $Q(Y)$ совпадает с подпространством $L_{1}\left(M_{2}\right)$.

Итак, $Q(Y)$ - подпространство и $Y+Q(Y)=L_{1}\left(M_{1}\right)+L_{1}\left(M_{2}\right)=L_{1}(M)$. Таким образом, оба условия леммы 4 вьполнены, и $Y$ - чебышевскоеподпространство с линейным оператором $P_{Y}$. Теорема доказана.

Разумеется, чебышевские подпространства в $L_{1}(M)$ не исчерпьваются указанньми в теореме 1. Приведем простейший пример.

Пусть $M$ - множество из $k$ точек, $\sigma$-алгебра $\Sigma$ состоит из всех подмножеств $M$, а мера $\mu$ множества $E \in \Sigma$ равна количеству точек в $E$. Соответствующее пространство функций $L_{1}(M, \Sigma, \mu)$ обозначим через $\ell_{1}^{k}$. При $k=3$ пространство $\ell_{1}^{3}$ изоморфно трехмерному пространству $\mathbb{R}^{3}$, в котором норма задается единичным "шаром" - правильным октаэдром с вершинами на осях координат. Вектор $(1,1,1)$ не параллелен ни одной из граней октаэдра, поэтому порожденное этим вектором одномерноеподпространство является чебышевским. Очевидно, что это подпространство никак не представимо в виде, указанном в теореме 1 .

Этот пример можно распространить на пространство функций, суммируемых на отрезке по мере Лебега. Следуя Моррису [3], положим

$$
Y=\left\{g \in L_{1}[0,3]: g(x)=g(x+1)=g(x+2) \text { для почти всех } x \in[0,1]\right\} \text {. }
$$

Подпространство $Y$, очевидно, не принадлежит к классу подпространств из теоремы 1. Тот факт, что оно является чебьшевским, непосредственно доказан в [3]. В то же время этот факт вытекает из следующей общей теоремы о “перенесении" чебышевских подпространств пространства $\ell_{1}^{k}$ в произвольное пространство $L_{1}(M)=L_{1}(M, \Sigma, \mu)$.

Теорема 2. Пусть $M=M_{1} \cup \cdots \cup M_{k}, \mu\left(M_{i} \cap M_{j}\right)=0$ nрu $i \neq j$ u при этом мнохества $M_{j}$ одинаковы в том смысле, что для всякого $j \geqslant 2$ существует взаимно однозначное отображсение $f_{j}: M_{1} \rightarrow M_{j}$, сохраняющее меру $\mu$. Пусть $Y_{0}-$ чебышевское подпространство в $\ell_{1}^{k}$. Тогда подпространство $Y=\left\{y \in L_{1}(M)\right.$ : $\left(y(t), y\left(f_{2}(t)\right), \ldots, y\left(f_{k}(t)\right)\right) \in Y_{0}$ для почти всех $\left.t \in M_{1}\right\}$ чебышевское в $L_{1}(M)$. 
ДокАЗАТЕльство. Каждому элементу $x \in L_{1}(M)$ и каждому $t \in M_{1}$ соответствует $k$-мерньй вектор $x_{t}=\left(x(t), x\left(f_{2}(t)\right), \ldots, x\left(f_{k}(t)\right)\right)$. Покажем, что

$$
Q(Y)=\left\{n \in L_{1}(M): n_{t} \in Q\left(Y_{0}, \ell_{1}^{k}\right) \text { для почти всех } t \in M_{1}\right\} .
$$

Пусть для некоторого $n \in Q(Y)$ множество $A$ тех точек $t \in M_{1}$, для которых $n_{t} \notin Q\left(Y_{0}\right)$, имеет положительную меру. Положим $y_{t}=P_{Y_{0}}\left(n_{t}\right), t \in M_{1}$. Зададим функцию $y: M \rightarrow \mathbb{R}$ равенством

$$
\left(y(t), y\left(f_{2}(t)\right), \ldots, y\left(f_{k}(t)\right)\right)=y_{t}, \quad t \in M_{1} .
$$

Поскольку $P_{Y_{0}}-$ непрерьвное отображение, функция $y$ измерима. С другой стороны, $\left\|n_{t}-y_{t}\right\|<\left\|n_{t}\right\|$ при $t \in A$ (здесь и далее норма берется в $\ell_{1}^{k}$ ), и для любого достаточно малого $\varepsilon>0$ найдется множество $A_{\varepsilon} \subset A$ положительной меры $\mu$ такое, что $\left\|n_{t}-y_{t}\right\|<(1-\varepsilon)\left\|n_{t}\right\|$ при $t \in A_{\varepsilon}$. Отсюда

$$
\begin{aligned}
& \int_{M}|n-y| d \mu=\int_{M_{1}}\left(\sum_{j=1}^{k}\left|n\left(f_{j}(t)\right)-y\left(f_{j}(t)\right)\right|\right) d \mu(t)=\int_{M_{1}}\left\|n_{t}-y_{t}\right\| d \mu(t) \\
& \quad=\int_{M_{1} \backslash A_{\varepsilon}}\left\|n_{t}-y_{t}\right\| d \mu(t)+\int_{A_{\varepsilon}}\left\|n_{t}-y_{t}\right\| d \mu(t) \\
& \quad \leqslant \int_{M_{1} \backslash A_{\varepsilon}}\left\|n_{t}\right\| d \mu(t)+(1-\varepsilon) \int_{A_{\varepsilon}}\left\|n_{t}\right\| d \mu(t)<\int_{M_{1}}\left\|n_{t}\right\| d \mu(t)=\int_{M}|n| d \mu .
\end{aligned}
$$

Мы получили, что, во-первых, функция $y$ суммируема (значит, $y \in Y$ ), во-вторых, $y$ ближе к $n$, чем нулевой элемент. Отсюда следует, что $n \notin Q(Y)$, это противоречит предположению.

Пусть теперь не тождественная нулю функция $n \in L_{1}(M)$ такова, что $n_{t} \in Q\left(Y_{0}\right)$ для почти всех $t \in M_{1}$. Тогда для любого $y \in Y, y \not \equiv 0$, имеем

$$
\int_{M}|n-y| d \mu=\int_{M_{1}}\left\|n_{t}-y_{t}\right\| d \mu(t)>\int_{M_{1}}\left\|n_{t}\right\| d \mu(t)=\int_{M}|n| d \mu,
$$

где строгость неравенства обосновывается так же, как выше.

Итак, (1) доказано. При этом мы установили, что для всякого $n \in Q(Y)$ справедливо равенство $P_{Y}(n)=\{0\}$. Осталось доказать, что $Y+Q(Y)=L_{1}(M)$ (см. лемму 1). С этой целью для всякой функции $x \in L_{1}(M)$ возьмем функцию $y: M \rightarrow \mathbb{R}$ такую, что $y_{t}=P_{Y_{0}}\left(x_{t}\right)$ при всех $t \in M_{1}$. Поскольку оператор $P_{Y_{0}}$ непрерывен и $\left\|y_{t}\right\| \leqslant\left\|x_{t}-y_{t}\right\|+\left\|x_{t}\right\| \leqslant 2\left\|x_{t}\right\|$, функция $y$ измерима и суммируема, т.е. $y \in Y$. При этом $x-y \in Q(Y)$. Теорема доказана.

Теоремы 1 и 2 дают достаточно большой запас чебьшевских подпространств в $L_{1}(M)$, имеющих бесконечную размерность и бесконечную коразмерность. Общая задача описания таких подпространств представляется весьма сложной, если вообще разрешимой (по поводу чебышевских подпространств конечной размерности или коразмерности см. замечание 1).

В пространстве $L_{1}[0,1]$ чебышевских подпространств конечной ненулевой размерности или коразмерности вообще нет [8]. В связи с этим возникает вопрос: существует ли в $L_{1}[0,1]$ чебьшевское подпространство, отличное от описанных в теоремах 1 и 2 ? 
2. Пространство $C$. Далее через $C[K]$ будем обозначать пространство вещественнозначных непрерьвных функций на компактном хаусдорфовом топологическом пространстве $K$ (в современной терминологии $K$ - компакт, в терминологии [9] $K$ - бикомпакт) с обычной равномерной нормой $\|f\|=\sup \{|f(t)|: t \in K\}$.

Каждая функция $f \in C[K]$ достигает своей нормы на непустом замкнутом подмножестве $m(f):=\{t \in K:|f(t)|=\|f\|\}$. О чебьшевских подпространствах в $C[K]$ см. [1], [2], [5]. Здесь мы докажем следующее утверждение.

Теорема 3. Oператор метрического проектирования $P_{Y}$ на нетривиальное собственное чебышевское подпространство $Y \subset C[K]$ линеен тогда и только тогда, когда $Y$ имеет в $C[K]$ коразмерность 1.

ДокАЗАТЕЛЬСтво. Достаточность следует из леммы 3.

Необходимость. Далее мы будем неоднократно пользоваться следующим легко проверяемым фактом:

(*) если элемент $n \in C[K]$ квазиортогонален к подпространству $Y \subseteq C[K]$, то и любой элемент $n^{\prime} \in C[K]$ с $m\left(n^{\prime}\right) \supseteq m(n), \operatorname{sign} n^{\prime}(t)=\operatorname{sign} n(t)$ при $t \in m(n)$, также квазиортогонален к $Y$.

Предположим, вопреки утверждению теоремы, что для некоторого нетривиального собственного чебьшевского подпространства $Y \subset C[K]$ коразмерности $>1$ оператор $P_{Y}$ линеен. Тогда по лемме 2 квазиортогональное множество $Q(Y)$ является линейным подпространством.

Поскольку $\operatorname{codim} Y>1$ и $Y+Q(Y)=C[K]$ (см. лемму 1$)$, в $Q(Y)$ найдутся два линейно независимых элемента $n_{1}$ и $n_{2}$ с $\left\|n_{1}\right\|=\left\|n_{2}\right\|=1$.

Мы можем считать, что $m\left(n_{1}\right) \cap m\left(n_{2}\right)=\varnothing$ (иначе вместо $n_{1}$ и $n_{2}$ можно взять $\left(n_{1}+n_{2}\right) /\left\|n_{1}+n_{2}\right\|$ и $\left(n_{1}-n_{2}\right) /\left\|n_{1}-n_{2}\right\|$, так как при $m\left(n_{1}\right) \cap m\left(n_{2}\right) \neq \varnothing$, очевидно, $m\left(n_{1}+n_{2}\right) \cap m\left(n_{1}-n_{2}\right)=\varnothing$, в то же время в силу линейности $\left.Q(Y) n_{1} \pm n_{2} \in Q(Y)\right)$.

Можем считать также, что $K \neq m\left(n_{1}\right) \cup m\left(n_{2}\right)$. В противном случае элементы $n_{1}$ и $n_{2}$ можно изменить так, чтобы они этому свойству удовлетворяли. Действительно, если $m\left(n_{1}\right) \cap m\left(n_{2}\right)=\varnothing$ и $K=m\left(n_{1}\right) \cup m\left(n_{2}\right)\left(m\left(n_{1}\right)\right.$ и $m\left(n_{2}\right)$ - компакты $)$, то пространство $C[K]$ изометрически изоморфно прямому произведению $C\left[m\left(n_{1}\right)\right] \times C\left[m\left(n_{2}\right)\right]$ с нормой $\left\|\left(f_{1}, f_{2}\right)\right\|=\max \left\{\left\|f_{1}\right\|,\left\|f_{2}\right\|\right\}$; здесь $f_{i} \in C\left[m\left(n_{i}\right)\right], i=1,2 ;\left(f_{1}, f_{2}\right) \in C\left[m\left(n_{1}\right)\right] \times$ $C\left[m\left(n_{2}\right)\right]$. Обозначим через $n_{i i}$ сужение функции $n_{i}$ на компакт $m\left(n_{i}\right), i=1,2$. Обе функции $\left(n_{11}, 0\right)$ и $\left(0, n_{22}\right)$ лежат в $Q(Y)$ согласно $(*)$. Ввиду нетривиальности подпространства $Y$ существует не тождественная нулю функция $y=\left(y_{1}, y_{2}\right) \in Y$. Тогда одна из двух компонент, например $y_{1}$, не тождественна нулю. Будем считать $\left\|y_{1}\right\|=1$. При этом $m\left(y_{1}+n_{11}\right) \neq m\left(n_{1}\right)$, иначе $y_{1}= \pm n_{11}$, и при достаточно малых положительных $\lambda$ имеем $\left\|\left(n_{11}, 0\right) \mp \lambda\left(y_{1}, y_{2}\right)\right\|<\left\|\left(n_{11}, 0\right)\right\|$, что невозможно, так как $\left(n_{11}, 0\right) \in Q(Y)$. $\mathrm{C}$ другой стороны, $\left(y_{1}+n_{11}, 0\right) \in Q(Y)$, поскольку $\left(y_{1}+n_{11}, 0\right)=\left(y_{1},-n_{22}\right)+\left(n_{11}, n_{22}\right)$ и обе функции $\left(y_{1},-n_{22}\right)$ и $\left(n_{11}, n_{22}\right)$ лежат в $Q(Y)$ (первая - ввиду того что $\left\|y_{1}\right\|=$ $\left\|n_{22}\right\|=1$ и $\left(0, n_{22}\right) \in Q(Y)$, вторая - ввиду линейности $\left.Q(Y)\right)$. Таким образом, функции $\bar{n}_{1}=\left(y_{1}+n_{11}, 0\right)$ и $\bar{n}_{2}=\left(0, n_{22}\right)$ обладают следующими свойствами: $\bar{n}_{1}, \bar{n}_{2} \in Q(Y)$, $m\left(\bar{n}_{2}\right)=m\left(n_{2}\right), m\left(\bar{n}_{1}\right) \subset m\left(n_{1}\right)$ (причем последнее включение строгое). Следовательно, $K \neq m\left(\bar{n}_{1}\right) \cup m\left(\bar{n}_{2}\right)$.

Итак, с самого начала можно выбрать $n_{1}, n_{2} \in Q(Y)$ с $\left\|n_{1}\right\|=\left\|n_{2}\right\|=1$ такие, что множества $m\left(n_{1}\right)$ и $m\left(n_{2}\right)$ не пересекаются и их объединение не совпадает со всем ком- 
пактом $K$. Покажем, что эти элементы всегда можно изменить так, чтобы они удовлетворяли, помимо всех перечисленных условий, еще и следующему: $m\left(n_{1}+n_{2}\right) \cap\left(m\left(n_{1}\right) \cup\right.$ $\left.m\left(n_{2}\right)\right)=\varnothing$.

Поскольку $K$ является нормальным топологическим пространством [9, с. 244], по лемме П. С. Урысона [9, с. 172] существует функция $f \in C[K]$ со свойствами: $f(t) \equiv 0$ на $m\left(n_{1}\right), f(t) \equiv 1$ на $m\left(n_{2}\right)$ и $0 \leqslant f(t) \leqslant 1$ при всех $t \in K$. Положим $n_{2}^{\prime}=f n_{2}$, $n_{1}^{\prime}=(1-f) n_{1}$. Очевидно, $n_{i}^{\prime} \in Q(Y)$ согласно $(*), m\left(n_{i}^{\prime}\right)=m\left(n_{i}\right)$ при $i=1,2$ и, кроме того, $n_{i}^{\prime}(t)=0$ при $t \in m\left(n_{j}^{\prime}\right)$ для $j \neq i$.

Возьмем какую-нибудь точку $t_{0} \in K \backslash\left(m\left(n_{1}^{\prime}\right) \cup m\left(n_{2}^{\prime}\right)\right)$ и содержащее ее открытое множество $U=\left\{t \in K: n_{i}^{\prime}\left(t_{0}\right)-\varepsilon<n_{i}^{\prime}(t)<n_{i}^{\prime}\left(t_{0}\right)+\varepsilon, i=1,2\right\}$, где $\varepsilon \in(0,1 / 4)$ выбрано так, чтобы вьполнялось $U \cap\left(m\left(n_{1}^{\prime}\right) \cup m\left(n_{2}^{\prime}\right)\right)=\varnothing$. По лемме Урысона существует функция $g \in C[K]$, равная 1 в $t_{0}$, равная 0 на $K \backslash U$ и при всех $t \in K$ удовлетворяющая неравенствам $0 \leqslant g(t) \leqslant 1$.

Положим $n_{i}^{\prime \prime}=n_{i}^{\prime}+\left(3 / 4-n_{i}^{\prime}\left(t_{0}\right)\right) g, i=1,2$. Нетрудно показать, что $\left\|n_{i}^{\prime \prime}\right\|=1$. Действительно, $n_{i}^{\prime \prime}(t)=n_{i}^{\prime}(t)$ при $t \in K \backslash U$, а при $t \in U n_{i}^{\prime \prime}(t) \subseteq\left[\min \left\{3 / 4, n_{i}^{\prime}\left(t_{0}\right)\right\}-\varepsilon\right.$, $\left.\max \left\{3 / 4, n_{i}^{\prime}\left(t_{0}\right)\right\}+\varepsilon\right] \subset(-1,1)$ в силу выбора числа $\varepsilon$. Легко проверить, что $m\left(n_{i}^{\prime \prime}\right)=$ $m\left(n_{i}^{\prime}\right)=m\left(n_{i}\right), n_{i}^{\prime \prime} \in Q(Y), n_{i}^{\prime \prime}\left(t_{0}\right)=3 / 4$ и, кроме того, $n_{i}^{\prime \prime}(t)=0$ при $t \in m\left(n_{j}^{\prime \prime}\right)$ для $j \neq i$.

В точке $t_{0}$ вьполнено $n_{1}^{\prime \prime}+n_{2}^{\prime \prime}=3 / 2$, поэтому $\left\|n_{1}^{\prime \prime}+n_{2}^{\prime \prime}\right\| \geqslant 3 / 2$. С другой стороны, $\left|n_{1}^{\prime \prime}(t)+n_{2}^{\prime \prime}(t)\right|=1$ при $t \in m\left(n_{1}^{\prime \prime}\right) \cup m\left(n_{2}^{\prime \prime}\right)$. Отсюда следует, что $m\left(n_{1}^{\prime \prime}+n_{2}^{\prime \prime}\right) \cap\left(m\left(n_{1}^{\prime \prime}\right) \cup\right.$ $\left.m\left(n_{2}^{\prime \prime}\right)\right)=\varnothing$.

Итак, с самого начала можно выбрать элементы $n_{1}, n_{2} \in Q(Y)$ со свойствами: $\left\|n_{1}\right\|=$ $\left\|n_{2}\right\|=1, m\left(n_{1}+n_{2}\right) \cap\left(m\left(n_{1}\right) \cup m\left(n_{2}\right)\right)=\varnothing$.

Нам понадобится

ЛЕмма 6 [2]. Если $Y$ - чебышевское подпространство в $C[K]$ u $n \in Q(Y)$, то всякая не тождественная нулю функиия $y \in Y$ не обращается в нуль тохдественно на множестве $m(n)$.

Мы предположили, что $Q(Y)$ - линейное подпространство. Следовательно, $n_{1}+n_{2} \in$ $Q(Y)$. Согласно лемме 6 найдутся точка $\tau \in m\left(n_{1}+n_{2}\right)$ и функция $y \in Y$ такие, что $y(\tau)>0$. Без ограничения общности можно считать, что и $\left(n_{1}+n_{2}\right)(\tau)>0$.

Для точки $\tau$ выберем окрестность $V$ так, чтобы при $t \in V$ вьполнялись неравенства $y(t)>y(\tau) / 2,\left(n_{1}+n_{2}\right)(t)>0$ и чтобы замькание $\bar{V}$ не пересекалось с множествами $m\left(n_{1}\right)$ и $m\left(n_{2}\right)$. Вновь, пользуясь леммой Урысона, возьмем функцию $h \in C[K]$ со свойствами: $h(\tau)=1, h(t) \equiv 1-\varepsilon$ при $t \in K \backslash V$ и $1-\varepsilon \leqslant h(t) \leqslant 1$ при всех $t \in K$. При этом $\varepsilon \in(0,1)$ выберем столь малым, чтобы выполнялись равенства $\left\|h \cdot n_{i}\right\|=1-\varepsilon$ и $m\left(h \cdot n_{i}\right)=m\left(n_{i}\right), i=1,2$.

Тогда $h \cdot n_{i} \in Q(Y)$ согласно $(*)$, откуда $n:=h \cdot\left(n_{1}+n_{2}\right) \in Q(Y)$ в силу линейности $Q(Y)$. С другой стороны, поскольку $m(n) \subset\left(V \cap m\left(n_{1}+n_{2}\right)\right)$, то $n(t)>0$ и $y(t)>y(\tau) / 2>0$ при $t \in m(n)$. Отсюда $\|n-\lambda y\|<\|n\|$ при достаточно малых положительных $\lambda$, так что функция $n$ не может быть квазиортогональной к подпространству $Y$. Полученное противоречие приводит к тому, что предположение о линейности $Q(Y)$ неверно. Теорема доказана.

В качестве примера подпространства из теоремы 3 возьмем подпространство $Y:=$ $\left\{f \in C[0,1]: \int_{0}^{1} f(t) d t=0\right\}$. Очевидно, $Y$ имеет коразмерность 1 в $C[0,1]$. Нетрудно 
проверить, что $Q(Y)$ состоит из постоянных функций, так что по лемме $4 Y$ - чебышевскоеподпространство. Оператор метрического проектирования на это подпространство действует так: $P_{Y}(f)(t)=f(t)-\int_{0}^{1} f(\tau) d \tau$, т.е. является линейным оператором.

Автор благодарен Е. П. Долженко и С. В. Конягину за внимание к работе.

\section{СПИСОК ЦИТИРОВАННОЙ ЛИТЕРАТУРЫ}

[1] Singer I. Best Approximation in Normed Linear Spaces by Elements of Linear Subspaces. Bucharest: Publ. House Acad. R.S. România, 1970; Berlin-Heidelberg-New York: Springer, 1970.

[2] Cheney E. W., Wulbert D. E. The existence and unicity of best approximations // Math. Scand. 1969. V. 24. №1. P. 113-140.

[3] Morris P. D. Chebyshev subspaces of $L^{1}$ with linear metric projection // J. Approx. Theory. 1980. V. 29. № 3. P. 231-234.

[4] Данфорд Н., Шварц Дж. Линейные операторы. Общая теория. М., 1962.

[5] Гаркави А. Л. Теория наилучшего приближения в линейных нормированных пространствах // Итоги науки. Математический анализ. М.: ВИНИТИ, 1969. С. 75-132.

[6] Phelps R. R. Čebyshev subspaces of finite dimension in $L_{1} / /$ Proc. Amer. Math. Soc. 1966. V. 17. № 3. P. 646-652.

[7] Гаркави А. Л. Характеристика чебьшевских подпространств конечной коразмерности в $L_{1}$ // Матем. заметки. 1970. Т. 7. № 2. С. 155-163.

[8] Phelps R.R. Uniqueness of Hahn-Banach extensions and unique best approximation // Trans. Amer. Math. Soc. 1960. V. 95. № 2. P. 238-255.

[9] Александров П. С. Введение в теорию множеств и общую топологию. М.: Наука, 1977.

Московский государственный университет им. М.В. Ломоносова

Поступило 13.05.96

Исправленный вариант

05.03 .97 